\title{
Perfusion and permeability MRI in glioma grading
}

Sonay Aydin ${ }^{1,2^{*}}$, Erdem Fatihoğlu ${ }^{1}$ (D), Pınar Nercis Koşar ${ }^{1}$ (D) and Elif Ergün ${ }^{1}(\mathbb{D})$

\begin{abstract}
Background: MRI is successful in showing the anatomy of probable pathologies of the central nervous system. Although it may not be sufficient to reveal physiological and metabolic changes, advanced MRI techniques, such as perfusion and permeability MRI, are the key to overcoming these limitations. The aim of this study was to detect the efficacy of permeability and perfusion MRI techniques.

Results: The study included 38 patients with a pathology result of primary brain glioma. The permeability MRI (Ktrans, Ve), perfusion MRI values (CBV, CBF), and pathology results were evaluated. The high-grade group included 22 patients, and the low-grade group, 16 patients. Mean CBV and CBF, median Ktrans, and Ve values were higher in the high-grade group. All parameters tended to elevate with grade and had a positive correlation. CBV $>2.25$, with sensitivity and specificity of $100 \%$, CBF $>2.02$, with sensitivity and specificity of $100 \%$, Ktrans $>0.043$, with sensitivity of $81.82 \%$ and specificity of $100 \%$, and $V e>0.255$, with sensitivity and specificity of $100 \%$, can predict high grade.
\end{abstract}

Conclusion: Perfusion and permeability MRI can be used safely for the differentiation of high- and low-grade gliomas and for the prediction of glioma grades.

Keywords: Permeability, MRI, Glioma, Grading

\section{Background}

Brain tumors, whether primary or metastatic, are commonly encountered, and their prognosis is strongly related with their grade. Gliomas are the most common primary brain tumor in adults [1].

Magnetic resonance imaging (MRI) is the current primary method to identify the anatomic details of the central nervous system (CNS). However, it is not so successful in showing physiological and metabolic changes [2]. Advanced MRI techniques are the solution for this problem. Of these techniques, perfusion MRI and permeability MRI are the most commonly used [3].

Perfusion MRI provides information about tissue vascularization, in vivo tumor angioneogenesis, and microcirculation. Perfusion MRI offers information about tissue blood volume, blood flow, and oxygenation of tissues. The signal changes in arteries/veins, which occur with the passage of paramagnetic contrast agent

\footnotetext{
* Correspondence: sonaydin89@hotmail.com

${ }^{1}$ Department of Radiology, Ankara Training and Research Hospital, Ankara, Turkey

${ }^{2}$ Department of Radiology, Sami Ulus Training and Research Hospital, Altindag, 06340 Ankara, Turkey
}

are used to create relative cerebral blood volume (rCBV), mean transit time (MTT), and cerebral blood flow (rCBF) parameters [4].

Neoangiogenesis and vascular proliferation are important parameters in deciding the grade of gliomas. Highgrade gliomas tend to have increased angiogenesis, and neoangiogenesis makes the blood-brain barrier (BBB) more permeable. The changes in vascular permeability cause an increase in extravasations of contrast medium and changes in $\mathrm{rCBV}, \mathrm{rCBF}$, and MTT values [5].

Permeability MRI is a relatively new method in comparison with perfusion MRI. It is used to estimate vascular permeability by measuring contrast medium leakage through the BBB. It has two main parameters: (1) vascular transfer constant (Ktrans), which describes the permeability of blood vessels to contrast medium based on a two-compartment pharmacokinetic model, considering the shape of the uptake and wash-out of the contrast agent. (2) Extravascular and extracellular volume fraction (Ve) is the interstitial volume in which extracellular contrast agents may accumulate if they cross the BBB (expressed in milliliter of extravascular extracellular volume $/ 100 \mathrm{ml}$ of tissue or percentage) [6]. 
Grade is an important parameter for the prognosis and treatment of brain tumors, as clinicians generally demand to know the exact grade before performing surgery [3]. The aim of this study was to evaluate the performance and success of perfusion and permeability MRI methods in grading gliomas and discriminating high- and low-grade tumors.

\section{Methods}

Approval for the study was granted by the Institutional Ethics Review Board. The study was performed between August 2016 and August 2017.

This retrospective study included patients with a diagnosis of primary brain tumor confirmed with pathology and who had undergone a permeability and perfusion MRI study before the surgery. A total of 65 patients were evaluated and 27 were excluded as they did not have permeability or perfusion MRI studies. Thus, 38 patients were evaluated.

Permeability MRI (Ktrans, Ve), perfusion MRI (rCBV, $\mathrm{rCBF}$ ) parameters, and the pathological diagnosis were recorded together with age and gender data.

The pathology results were classified according to the WHO grading system: low-grade (grades 1 and 2) and high grade (grades 3 and 4). The diagnostic success of the perfusion and permeability MRI methods was evaluated for the high-grade and low-grade groups and for every subgroup: grades $1-4$. Grade 1 and 2 subgroups had to be combined and analyzed as a single group as there were so few patients in the grade 1 subgroup.

All the MRI examinations were performed using 1.5 Tesla MRI systems (Magnetom Aera, Siemens Healthcare $\mathrm{GmbH}$, Erlangen, Germany).

A gadolinium (Gd)-based contrast agent (Gadovist, Bayer) was administered in two, one-half boluses at a standard dose of $0.1 \mathrm{mmol} / \mathrm{kg}$ for permeability MRI first, and perfusion MRI secondarily.

Permeability MRI consists of two parts: the data sets for calculation of T1 maps and for production of T1weighted dynamic data. The T1 maps were created using three-dimensional volumetric interpolated breath-hold sequence [VIBE; $5.1 \mathrm{~ms}$ TR/1.8 ms TE, 2- and 15-flip angle, number of excitations (NEX) $1 / 48$ and 6, 4-mm- section thickness, 250-250-mm FOV, 192-138-mm matrix size]. To acquire the T1-weighted dynamic data set VIBE (4.3 ms TR/1.5 ms TE, 15-flip angle, NEX 1/4 1, 4-mm-section thickness, 250-250-mm FOV, 192-138$\mathrm{mm}$ matrix size, number of phases $1 / 4$ 50) was also performed.

Perfusion MRI, consists of dynamic T2*-weighted images, acquired using a gradient-echo echo-planar imaging pulse sequence $(1880 \mathrm{~ms}$ TR/30 ms TE, 90-flip angle, NEX 1/4 1, 5-mm-section thickness, 25 sections, 230-230-mm FOV, 128-128-mm matrix size).

Perfusion and permeability maps and numeric data were created using the tissue 4D program (Siemens Healthcare, Erlangen, Germany).

For permeability and perfusion MRI analysis, three regions of interest (ROIs) were used for each map, and the highest ROI value was selected to minimize selection bias. The size of the ROIs was kept constant (approximately $3 \mathrm{~mm}^{2}$ ) to maintain homogeneity. T1-weighted contrast-enhanced images were used for placement of ROIs in order to avoid necrotic and cystic tumor parts. Some examples of the cases can be seen in Figs. 1 and 2 .

\section{Statistical analysis}

Statistical analyses were performed using SPSS Statistics software (version 21.0; SPSS Inc., Chicago, IL, USA). Continuous parameters were stated as mean \pm standard deviation, and skewed continuous parameters were evaluated with the Mann-Whitney $U$ test. Categorical data were stated as frequencies $(n)$ and percentages (\%) and were compared using the Fisher exact or chi-square tests where appropriate. The Student's $t$ test and the MannWhitney $U$ test were used to analyze numerical variables in two groups. For more than two groups, ANOVA and the Kruskall-Wallis $H$ test were applied. For post hoc analysis, the Bonferroni test was used for ANOVA, and Dunn's test for the Kruskall-Wallis $H$ test. Pearson and Spearman correlation tests were used to define the relationships between numerical variables. The power and success of diagnostic tests were determined using ROC curve analysis and were expressed with positive/negative predictive values, sensitivity, and specificity. Prediction values were calculated with the Youden index.

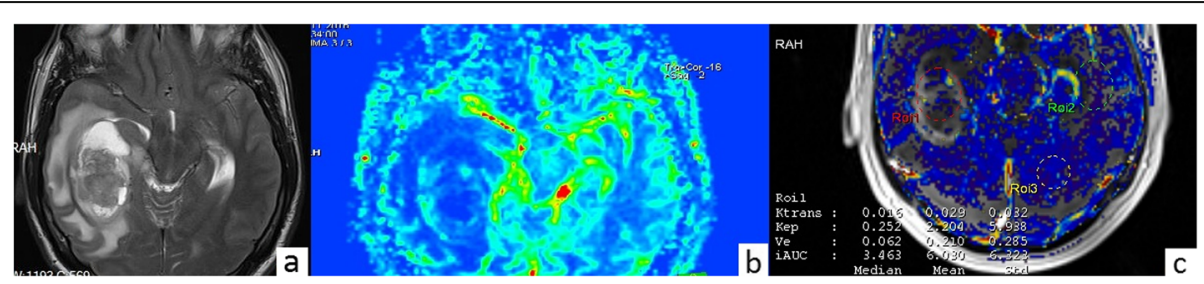

Fig. 1 A 44-year-old male. Heterogeneous mass with cystic component is seen on T2WI (a). There is no apparent increase in perfusion on rCBV map (b). Permeability parameters indicate low-grade glioma (Ktrans 0.029, Ve 0.210) (c). Pathological diagnosis is WHO grade 2 glioma (diffuse astrocytoma) 


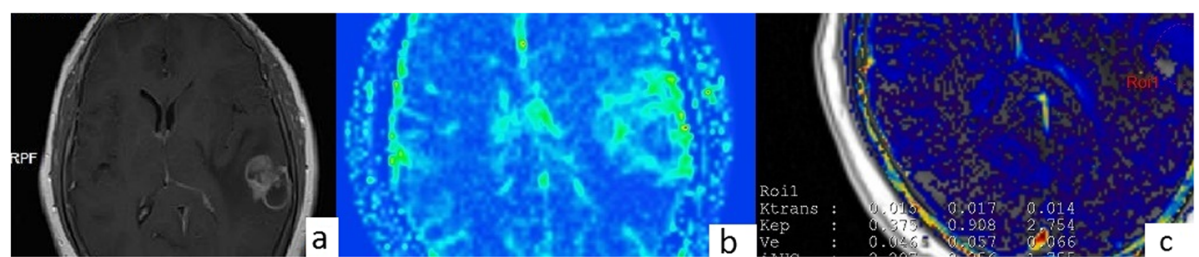

Fig. 2 A 60-year-old female. Heterogeneously enhancing mass in the left parietal lobe on T1WI axial image. Perilesional oedema is also present (a). There is an increased perfusion on rCBV map (b). Permeability data indicates low-grade glioma (Ktrans 0.017, Ve 0.057) (c). Pathological diagnosis is WHO grade 3 glioma (anaplastic astrocytoma)

A two-tailed value of $p<0.05$ was considered statistically significant.

\section{Results}

The study population comprised 38 patients; 8 patients with grade 4 glioma (21.1\%), 14 with grade 3 glioma (36.8\%), 14 with grade 2 glioma (36.8\%), and 2 with grade 1 glioma (5.3\%). There were 22 patients with highgrade glioma (57.9\%), and 16 patients with low-grade glioma (42.1\%).

The 38 patients comprised $22(57.9 \%)$ males and 16 (42.1\%) females with a mean age of $46.8 \pm 17.7$ years (range, 9-79 years).

rCBV values varied between 1.77-6.89 and mean 4.18 \pm 1.7. $\mathrm{rCBF}$ values varied between $1.62-7.00$ and mean $4.0 \pm 1$.6. Ktrans values varied between $0.01-0.2$, median 0.04. Ve values varied between $0.02-0.70$ and median 0.32 .

Mean age was seen to be higher in the high-grade group $(56.05 \pm 11.12$ years vs. $34.00 \pm 17.33$ years; $p<$ 0.001 ). The mean age was lower in the grade $1-2$ subgroups than in the grade 3 and 4 subgroups. No difference was determined between the grade 3 and 4 subgroups in respect of mean age (grades 1-2: $34.00 \pm$ 17.33 years vs. grade 3: $58.57 \pm 12.67$ years vs. grade 4 : $51.63 \pm 6.19$ years; $p<0.001)$. Gender distribution was not different amongst any subgroups (Table 1 ).

Table 1 Distribution of findings according to high- and lowgrade groups

\begin{tabular}{llll}
\hline & $\begin{array}{l}\text { High grade } \\
n=22(\%)\end{array}$ & $\begin{array}{l}\text { Low grade } \\
n=16(\%)\end{array}$ & $p$ \\
\hline Age & $56.05 \pm 11.12$ & $34 \pm 17.33$ & $<0.001^{*}$ \\
Gender & $10(45.5)$ & $6(37.5)$ & 0.744 \\
$\quad$ Male & $12(54.5)$ & $10(62.5)$ & \\
$\quad$ Female & $5.48 \pm 0.72$ & $2.41 \pm 0.55$ & $<0.001^{*}$ \\
rCBV & $5.10 \pm 1.21$ & $2.46 \pm 0.57$ & $<0.001^{*}$ \\
rCBF & $0.1(0.03-0.21)$ & $0.02(0.01-0.04)$ & $<0.001^{*}$ \\
Ktrans & $0.49(0.26-0.71)$ & $0.12(0.02-0.26)$ & $<0.001^{*}$ \\
Ve & & & \\
\hline${ }^{*} p<0.05$ statistical significance & &
\end{tabular}

${ }^{*} p<0.05$ statistical significance
Mean rCBV $(5.48 \pm 0.72$ vs. $2.41 \pm 0.55 ; p<0.001)$, mean $\mathrm{rCBF}(5.10 \pm 1.21$ vs. $2.46 \pm 0.57 ; p<0.001)$, median Ktrans ( 0.1 vs. $0.02 ; p<0.001)$, and median Ve $(0.49$ vs. $0.12 ; p<0.001)$ were higher in the high-grade group (Table 1).

Mean rCBV was higher in the grade 4 subgroup than in grade 3 , and higher in the grade 3 subgroup than in the grade 1-2 subgroups (grades 1-2: $2.41 \pm 0.55$ vs. grade $3: 5.03 \pm 0.35$ vs. grade $4: 6.27 \pm 0.45 ; p<0.001$ ) (Table 2).

Mean $\mathrm{rCBF}$ was higher in the grade 4 subgroup than in grade 3 and higher in grade 3 than grades 1-2 (grades $1-2: 2.46 \pm 0.57$ vs. grade $3: 4.33 \pm 0.58$ vs. grade 4 : $6.45 \pm 0.72 ; p<0.001$ ) (Table 2).

Median Ktrans was higher in the grade 4 subgroup than in grade 3 and higher in grade 3 than grades $1-2$ (grades $1-2: 0.02$ vs. grade 3: 0.08 vs. grade 4: $0.17 ; p<$ 0.001) (Table 2).

Median Ve was higher in the grade 4 subgroup than in grade 3 and higher in grade 3 than grade $1-2$ (grades $1-$ 2: 0.12 vs. grade 3 : 0.38 vs. grade $4: 0.70 ; p<0.001)$ (Table 2).

A positive correlation was determined between perfusion ( $\mathrm{rCBV}, \mathrm{rCBF}$ ) and permeability (Ktrans, Ve) parameters when the whole study population was considered. This positive correlation did not remain stable in every subgroup (Table 3 ).

\section{Predicting high-grade gliomas}

A rCBV value of $>3.25$ was found to predict a highgrade glioma with $100 \%$ specificity and sensitivity (AUC: 1.000; $p<0.001)$.

A rCBF value of $>3.22$ was found to predict a highgrade glioma with $100 \%$ specificity and sensitivity (AUC: $1.000 ; p<0.001$ ).

A Ktrans value of $>0.043$ was found to predict a highgrade glioma with $100 \%$ specificity and $81.82 \%$ sensitivity (AUC: $0.962 ; p<0.001$ ).

A Ve value of $>0.255$ was found to predict a highgrade glioma with $100 \%$ specificity and sensitivity (AUC: $1.000 ; p<0.001)$. 
Table 2 Distribution of findings according to grade subgroups

\begin{tabular}{|c|c|c|c|c|}
\hline & \multicolumn{3}{|l|}{ Grade } & \multirow[t]{2}{*}{$p$} \\
\hline & $\begin{array}{l}1-2 \\
n=16(\%)\end{array}$ & $\begin{array}{l}3 \\
n=14(\%)\end{array}$ & $\begin{array}{l}4 \\
n=8(\%)\end{array}$ & \\
\hline Age & $34.00 \pm 17.33$ & $58.57 \pm 12.67$ & $51.63 \pm 6.19$ & $<0.001$ \\
\hline \multicolumn{5}{|l|}{ Gender } \\
\hline Female & $6(37.5)$ & $6(42.9)$ & $4(50.0)$ & \multirow[t]{2}{*}{0.913} \\
\hline Male & $10(62.5)$ & $8(57.1)$ & $4(50.0)$ & \\
\hline rCBV & $2.41 \pm 0.55$ & $5.03 \pm 0.35$ & $6.27 \pm 0.45$ & $<0.001$ \\
\hline rCBF & $2.46 \pm 0.57$ & $4.33 \pm 0.58$ & $6.45 \pm 0.72$ & $<0.001$ \\
\hline Ktrans & $0.02(0.01-0.04)$ & $0.08(0.03-0.15)$ & $0.17(0.08-0.21)$ & $<0.001$ \\
\hline Ve & $0.12(0.02-0.26)$ & $0.38(0.26-0.67)$ & $0.70(0.38-0.71)$ & $<0.001$ \\
\hline
\end{tabular}

\section{Predicting grade 3 gliomas}

A rCBV value of $>3.25$ was found to predict a grade 3 glioma with $100 \%$ specificity and sensitivity (AUC: 1.000 ; $p<0.001$ ).

A rCBF value of $>3.22$ was found to predict a grade 3 glioma with $100 \%$ specificity and sensitivity (AUC: 1.000 ; $p<0.001)$.

A Ktrans value of $>0.038$ was found to predict a grade 3 glioma with $81.25 \%$ specificity and $92.86 \%$ sensitivity (AUC: $0.940 ; p<0.001$ ).

A Ve value of $>0.255$ was found to predict a grade 3 glioma with $100 \%$ specificity and sensitivity (AUC: 1.000 ; $p<0.001)$.

\section{Predicting grade 4 gliomas}

A rCBV value of $>5.67$ was found to predict a grade 4 glioma with $92.86 \%$ specificity and $100 \%$ sensitivity (AUC: $0.991 ; p<0.001$ ).

A $\mathrm{rCBF}$ value of $>5.44$ was found to predict a grade 4 glioma with $100 \%$ specificity and sensitivity (AUC: 1.000 ; $p<0.001)$.

A Ktrans value of $>0.111$ was found to predict a grade 4 glioma with $85.7 \%$ specificity and $75 \%$ sensitivity (AUC: $0.853 ; p<0.001$ ).

A Ve value of $>0.49$ was found to predict a grade 4 glioma with $78.57 \%$ specificity and $87.5 \%$ sensitivity (AUC: $0.884 ; p<0.001$ ) (Table 4).

Table 3 Correlations between variables in the whole population and grade subgroups

\begin{tabular}{|c|c|c|c|c|c|c|c|}
\hline & & \multicolumn{2}{|l|}{ rCBV } & \multicolumn{2}{|l|}{$\mathrm{rCBF}$} & \multicolumn{2}{|c|}{ Ktrans } \\
\hline & & $r$ & $p$ & $r$ & $p$ & $r$ & $p$ \\
\hline \multirow[t]{4}{*}{ Whole population } & CBV & - & - & 0.944 & $<0.001^{*}$ & 0.849 & $<0.001^{*}$ \\
\hline & $\mathrm{CBF}$ & 0.944 & $<0.001^{*}$ & - & - & 0.870 & $<0.001^{*}$ \\
\hline & Ktrans & 0.849 & $<0.001^{*}$ & 0.870 & $<0.001^{*}$ & - & - \\
\hline & Ve & 0.930 & $<0.001^{*}$ & 0.937 & $<0.001^{*}$ & 0.867 & $<0.001^{*}$ \\
\hline \multirow[t]{4}{*}{$1-2$} & CBV & - & - & 0.786 & $<0.001^{*}$ & 0.401 & 0.123 \\
\hline & $\mathrm{CBF}$ & 0.786 & $<0.001^{*}$ & - & - & 0.397 & 0.128 \\
\hline & Ktrans & 0.401 & 0.123 & 0.397 & 0.128 & - & - \\
\hline & Ve & 0.665 & $0.005^{*}$ & 0.671 & $0.004^{*}$ & 0.397 & 0.128 \\
\hline \multirow[t]{4}{*}{3} & CBV & - & - & 0.792 & $0.001^{*}$ & 0.357 & 0.210 \\
\hline & $\mathrm{CBF}$ & 0.792 & $0.001^{*}$ & - & - & 0.597 & $0.024^{*}$ \\
\hline & Ktrans & 0.357 & 0.210 & 0.597 & $0.024^{*}$ & - & - \\
\hline & Ve & 0.506 & 0.065 & 0.651 & $0.012^{*}$ & 0.757 & $0.002^{*}$ \\
\hline \multirow[t]{4}{*}{4} & CBV & - & - & 0.722 & $0.043^{*}$ & 0.167 & 0.693 \\
\hline & CBF & 0.722 & $0.043^{*}$ & - & - & 0.143 & 0.736 \\
\hline & Ktrans & 0.167 & 0.693 & 0.143 & 0.736 & - & - \\
\hline & Ve & 0.690 & 0.058 & 0.762 & $0.028^{*}$ & 0.262 & 0.531 \\
\hline
\end{tabular}


Table 4 Diagnostic value of the variables according to grade subgroups

\begin{tabular}{|c|c|c|c|c|c|c|c|}
\hline & Predictive Value & Sensitivity & Specificity & PPV & NPV & AUC & $p$ \\
\hline \multicolumn{8}{|c|}{ High grade (ref: low) } \\
\hline rCBV & $>3.25$ & 100.00 & 100.00 & 100.0 & 100.0 & 1.000 & $<0.001^{*}$ \\
\hline $\mathrm{rCBF}$ & $>3.22$ & 100.00 & 100.00 & 100.0 & 100.0 & 1.000 & $<0.001^{*}$ \\
\hline Ktans & $>0.043$ & 81.82 & 100.00 & 100.0 & 22.4 & 0.962 & $<0.001$ \\
\hline Ve & $>0.255$ & 100.00 & 100.00 & 100.0 & 100.0 & 1.000 & $<0.001^{*}$ \\
\hline \multicolumn{8}{|c|}{ Grade 3 (ref: 1, 2) } \\
\hline rCBV & $>3.25$ & 100.00 & 100.00 & 100.0 & 100.0 & 1.000 & $<0.001^{*}$ \\
\hline $\mathrm{rCBF}$ & $>3.22$ & 100.00 & 100.00 & 100.0 & 100.0 & 1.000 & $<0.001^{*}$ \\
\hline Kttrans & $>0.038$ & 92.86 & 81.25 & 98.9 & 37.4 & 0.940 & $<0.001^{*}$ \\
\hline Ve & $>0.255$ & 100.00 & 100.00 & 100.0 & 100.0 & 1.000 & $<0.001^{*}$ \\
\hline \multicolumn{8}{|c|}{ Grade 4 (ref: 1, 2) } \\
\hline rCBV & $>5.67$ & 100.00 & 92.86 & 99.6 & 100.0 & 0.991 & $<0.001^{*}$ \\
\hline $\mathrm{rCBF}$ & $>5.44$ & 100.00 & 100.00 & 100.0 & 100.0 & 1.000 & $<0.001^{*}$ \\
\hline Ktrans & $>0.111$ & 75.00 & 85.70 & 99.0 & 15.3 & 0.853 & $<0.001^{*}$ \\
\hline Ve & $>0.49$ & 87.50 & 78.57 & 98.7 & 24.9 & 0.884 & $<0.001^{*}$ \\
\hline
\end{tabular}

${ }^{*} p<0.05$ statistical significance

$P P V$ positive predictive value, NPV negative predictive value, AUC area under curve

\section{Discussion}

Gliomas are heterogeneous tumors. They can show a wide range of mitotic activity, cellular and nuclear pleomorphism, vascular proliferation, and necrotic content. These differences might be apparent even in different parts of the same tumor [7].

In literature, the success rates of conventional MRI in glioma grading range between $55 \%$ and $83.3 \%$. Classically, with the use of conventional MRI, tumor grade is predicted considering the presence of heterogeneity, necrosis, contour characteristics, and the presence of hemorrhage and diffusion restriction. However, it has been stated in the literature that these properties are not always reliable for the prediction of tumor grade [8]. Contrast enhancement is another parameter that correlates with tumor grade in conventional MRI techniques. However, it has been reported that approximately $40 \%$ of high-grade glioma cases might not enhance with gadolinium-based contrast medium $[9,10]$. In addition, low-grade gliomas might show avid contrast enhancement [11]. Reliable and correct grading on MRI is crucial for the treatment plan, especially for patients for whom biopsy is risky [12].

Neovascularization and micro vascular permeability are important parameters for glioma grading, and advanced MRI methods have been trying to provide information about these changes. Perfusion and permeability MRI techniques are the most commonly used for this purpose $[9,10]$.

Perfusion MRI provides information about tumoral neovascularization and capillary permeability, so that it can be effectively used as a tool for glioma grading. In literature, it has been stated that CBV values are correlated with tumor grade in both primary and metastatic brain tumors [13]. Prediction values suggested for rCBV to predict high-grade tumors differ between 1.50 and1.98, and the most widely used value is 1.75 [14-16]. The current study results confirmed the literature data, as all of the high-grade gliomas included in the study had $\mathrm{rCBV}$ values $>1.75$.

When 1.75 is used as the predictive value for $\mathrm{rCBV}$ to differentiate high- and low-grade gliomas, high-grade gliomas can be detected with sensitivity of $83 \%$ and specificity of $81 \%$. In contrast to the literature, according to the current study data, a predictive value of 3.25 can differentiate high-grade gliomas with a sensitivity and specificity of $100 \%$.

The mean rCBV value of the low-grade group was higher than the previous findings in literature (2.41 \pm 0.55 ). This difference could have been caused by the relatively small number of patients and the inclusion in the current study of oligodengroglioma cases. Although oligodendrogliomas are low-grade tumors, they generally have high rCBV values, because of their avid neovascularization $[13,17]$. It was seen to be consistent with the literature where oligodendroglioma cases have been included that high rCBV values $(4.42,5.01$, and 4.44$)$ were reported similar to high-grade gliomas (Fig. 3).

In the literature, the diagnostic success of rCBV has generally been examined for the differentiation of lowand high-grade gliomas. Unlike the literature, the predictive values indicating grade 3 and grade 4 subgroups were found to be different in the current study (a rCBV value of $>3.25$ predicts grade 3 with sensitivity and 


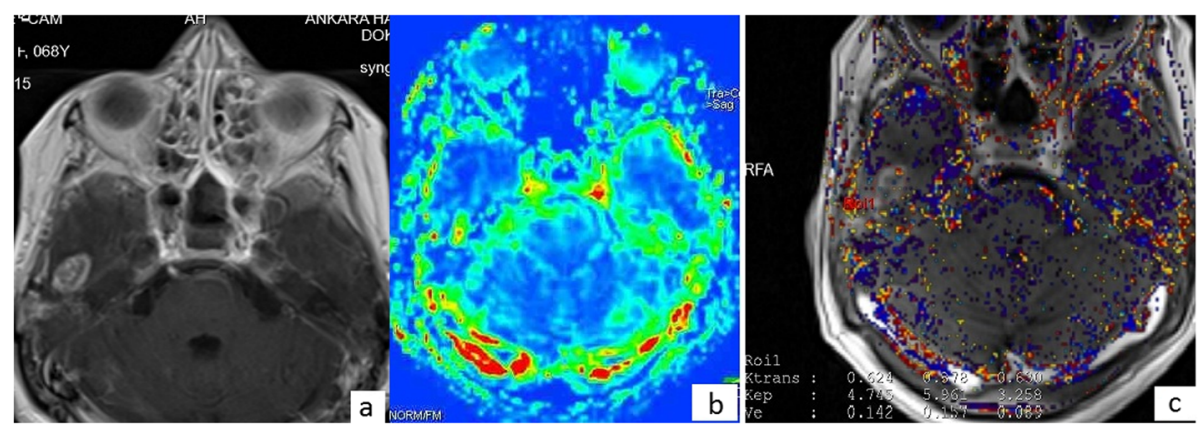

Fig. 3 A 68-year-old female. Enhancing mass in the right temporal lobe is seen on contrast enhanced T1WI (a, arrow). There is increased perfusion on rCBV map (rCBV 3.20) (b, arrow). Permeability data indicates high-grade according to Ktrans value (0.678) (c, arrow). Pathological diagnosis is WHO grade 2 oligodendroglioma

specificity of $100 \%$, and a rCBV value of $>5.67$ predicts grade 4 with sensitivity of $100 \%$, and specificity of 92.8\%). The current study can be considered to make a valuable contribution to the literature with these predictive values.

rCBF provides information about tissue perfusion. In the literature, it has been stated that $\mathrm{rCBF}$ values can differentiate low- and high-grade gliomas, but they are less reliable than $\mathrm{rCBV}$ values $[16,18,19]$. This is explained by the wide variability of $\mathrm{rCBF}$ values between different studies. This variability can be attributed to the heterogeneous nature of gliomas, such as neovascularisation and blood flow rates which might vary even in different parts of the same tumor [18]. In the current study, rCBF values seemed to be as successful as $\mathrm{rCBV}$ values. This difference could be attributed to the relatively homogeneous inner characteristics of the included glioma cases. Moreover, in other studies examining rCBF values, generally one ROI has been used, whereas three ROI placements were used in the current study, which might have minimized the heterogeneity of tumor vascularization and increased $\mathrm{rCBF}$ success. The use of at least 3 ROIs can be recommended when predicting tumor grade with $\mathrm{rCBF}$ values.

In the literature, the diagnostic success of $\mathrm{rCBF}$ has generally been examined for the differentiation of lowand high-grade gliomas. In the current study, unlike the literature, $\mathrm{rCBF}$ values were also found to be useful for the differentiation of grade $1-2$, grade 3 , and grade 4 subgroups from one another.

Permeability MRI is relatively a new method, and there is less knowledge about its success. It has been stated that Ktrans values increase together with the tumor grade [5]. The current study results are consistent with the literature, as the Ktrans values were found to be successful in differentiating high- and low-grade tumors. In the literature, there are few studies defining a predictive Ktrans value for the differentiation of low- and highgrade gliomas. Jain et al. suggested 0.045 as a predictive value [5]. In the current study, a Ktrans value of $>0.043$ was found to differentiate low- and high-grade gliomas (sensitivity $81.82 \%$, specificity $100 \%$ ).

Ve is another permeability MRI parameter. There is limited number of studies examining the diagnostic success of Ve in the differentiation of low- and high-grade gliomas $[14,19,20]$. However, no widely used predictive Ve value could be found in those studies. In the current study, consistent with the literature, Ve was determined to be useful in the differentiation of low- and high-grade gliomas. Furthermore, as a contribution to the literature, it was decided that the predictive Ve value of $>0.255$ could predict grade 3 glioma diagnosis with sensitivity and specificity of $100 \%$. A Ve value of $>0.49$ indicated grade 4 glioma with sensitivity of $87.5 \%$ and specificity of $78.57 \%$.

In the literature, studies have generally examined the correlation between perfusion MRI parameters ( $\mathrm{rCBV}$, rCBF, MTT, etc.). $\mathrm{rCBV}$ and $\mathrm{rCBF}$ values have been shown to have a positive correlation [16]. The current study results are consistent with the literature. Both in whole population and in all of the subgroups, rCBV and rCBF values had a positive correlation. No study could be found which gave information about the correlation between perfusion and permeability MRI parameters. According to the current study data, a positive correlation was present between all parameters in the whole study population. However, this correlation was not present in all the subgroups. Especially in high-grade gliomas, no correlation could be detected between perfusion and permeability parameters. It was concluded that in high-grade gliomas, the changes of blood flow (represented with perfusion parameters) and micro vascular permeability (represented with permeability parameters) do not happen in a balanced manner. Further studies on the molecular/tissue basis will be able to clarify the pathogenesis. It can be concluded that for more precise grading, especially in high-grade cases, combining permeability and perfusion techniques can increase accuracy. 
The study has some limitations. As the study focused on the differentiation of glioma grades, the performance of perfusion and permeability MRI could not be evaluated in the differentiation of tumoral and non-tumoral cases. The grade 1 and grade 2 subgroups had to be combined because of the small number of grade 1 glioma cases. Therefore, results cannot be defined independently for grade 1 and 2 tumors. The data of only one perfusion and permeability MRI examination were available for each case. Evaluation of follow-up results could have an effect on the performance of the methods.

\section{Conclusion}

Both perfusion and permeability MRI methods are sufficient and successful in the non-invasive differentiation of high- and low-grade gliomas. In addition, they can be used to differentiate grade 3 and grade 4 glioma cases. Especially in high-grade glioma cases, the use of both techniques could provide more accurate grading.

\section{Abbreviations}

BBB: Brain-blood barrier; CNS: Central nervous system; Ktarns: Vascular transfer constant; MRI: Magnetic resonance imaging; MTT: Mean transition time; rCBF: Relative cerebral blood flow; rCBV: Relative cerebral blood volume; ROI: Region of interest; Ve: Extravascular and extracellular volume fraction

\section{Acknowledgements}

Not applicable.

\section{Authors' contributions}

SA analyzed and interpreted the patient data. EF helped in writing the manuscript. PK helped in literatüre search and editing the manuscript. EE edited the last form of the manuscript. All authors read and approved the final manuscript.

\section{Funding}

Not applicable.

\section{Availability of data and materials}

The datasets used and/or analyzed during the current study are available from the corresponding author on reasonable request.

\section{Ethics approval and consent to participate}

All procedures performed in studies involving human participants were in accordance with the ethical standards of the institutional and/or national research committee and with the 1964 Helsinki Declaration and its later amendments or comparable ethical standards.

The local institutional review board approved the study (Ankara Training and Research Hospital institutional review board reference number: 0664-5557). A written informed consent was taken from all of the patients or from their parents if they were under the age of 18.

\section{Consent for publication}

Informed consent was acquired from all participants.

\section{Competing interests}

The authors declare that they have no competing interests.
Received: 20 December 2019 Accepted: 29 December 2019

Published online: 07 January 2020

\section{References}

1. Rebeles F, Fink J, Anzai Y, Maravilla KR (2006) Blood-brain barrier imaging and therapeutic potentials. Top Magn Reson Imaging 17(2):107-116. https:// doi.org/10.1097/RMR.0b013e31802f5df9

2. Aksoy FG, Lev MH (2000) Dynamic contrast-enhanced brain perfusion imaging: technique and clinical applications. Semin Ultrasound CT MR 21(6): $462-477$

3. Li X, Zhu Y, Kang H, Zhang Y, Liang H, Wang S, Zhang W (2015) Glioma grading by microvascular permeability parameters derived from dynamic contrast-enhanced MRI and intratumoral susceptibility signal on. susceptibility weighted imaging. Cancer Imaging 15:4. https://doi.org/10. 1186/s40644-015-0039-z

4. Law M, Young RJ, Babb JS, Peccerelli N, Chheang S, Gruber ML, Miller DC, Golfinos JG, Zagzag D, Johnson G (2008) Gliomas: predicting time to progression or survival with cerebral blood volume measurements at dynamic susceptibility-weighted contrast-enhanced perfusion MR imaging. Radiology 247(2):490-498. https://doi.org/10.1148/radiol.2472070898

5. Jain R, Ellika SK, Scarpace L, Schultz LR, Rock JP, Gutierrez J, Patel SC, Ewing J, Mikkelsen T (2008) Quantitative estimation of permeability surface-area product in astroglial brain tumors using perfusion $\mathrm{CT}$ and correlation with histopathologic grade. AJNR Am J Neuroradiol 29(4):694-700. https://doi. org/10.3174/ajnr.A0899

6. Cuenod CA, Balvay D (2013) Perfusion and vascular permeability: basic concepts and measurement in DCE-CT and DCE-MRI. Diagn Interv Imaging 94(12):1187-1204. https://doi.org/10.1016/j.diii.2013.10.010

7. Chawla S, Wang S, Wolf RL, Woo JH, Wang J, O'Rourke DM, Judy KD, Grady MS, Melhem ER, Poptani H (2007) Arterial spin-labeling and MR spectroscopy in the differentiation of gliomas. AJNR Am J Neuroradiol 28(9): 1683-1689. https://doi.org/10.3174/ajnr.A0673

8. Yang D, Korogi Y, Sugahara T, Kitajima M, Shigematsu Y, Liang L, Ushio Y, Takahashi M (2002) Cerebral gliomas: prospective comparison of multivoxel 2D chemical-shift imaging proton MR spectroscopy, echoplanar perfusion and diffusion-weighted MRI. Neuroradiology 44(8):656-666. https://doi.org/ 10.1007/s00234-002-0816-9

9. Spampinato MV, Smith JK, Kwock L, Ewend M, Grimme JD, Camacho DL, Castillo M (2007) Cerebral blood volume measurements and proton MR spectroscopy in grading of oligodendroglial tumors. AJR Am J Roentgenol 188(1):204-212. https://doi.org/10.2214/AJR.05.1177

10. Batra A, Tripathi RP, Singh AK (2004) Perfusion magnetic resonance imaging and magnetic resonance spectroscopy of cerebral gliomas showing imperceptible contrast enhancement on conventional magnetic resonance imaging. Australas Radiol 48(3):324-332. https://doi.org/10.1111/j.0004-8461. 2004.01315.x

11. Calli C, Kitis O, Yunten N, Yurtseven T, Islekel S, Akalin T (2006) Perfusion and diffusion MR imaging in enhancing malignant cerebral tumors. Eur J Radiol 58(3):394-403. https://doi.org/10.1016/j.ejrad.2005.12.032

12. Zonari P, Baraldi P, Crisi G (2007) Multimodal MRI in the characterization of glial neoplasms: the combined role of single-voxel MR spectroscopy, diffusion imaging and echo-planar perfusion imaging. Neuroradiology 49(10):795-803. https://doi.org/10.1007/s00234-007-0253-x

13. Hourani R, Horska A, Albayram S, Brant LJ, Melhem E, Cohen KJ, Burger $P C$, Weingart JD, Carson B, Wharam MD, Barker PB (2006) Proton magnetic resonance spectroscopic imaging to differentiate between nonneoplastic lesions and brain tumors in children. J Magn Reson Imaging 23(2):99-107. https://doi.org/10.1002/jmri.20480

14. Law M, Yang S, Wang H, Babb JS, Johnson G, Cha S, Knopp EA, Zagzag D (2003) Glioma grading: sensitivity, specificity, and predictive values of perfusion MR imaging and proton MR spectroscopic imaging compared with conventional MR imaging. AJNR Am J Neuroradiol 24(10):1989-1998

15. Danchaivijitr N, Waldman AD, Tozer DJ, Benton CE, Brasil Caseiras G, Tofts PS, Rees JH, Jager HR (2008) Low-grade gliomas: do changes in rCBV measurements at longitudinal perfusion-weighted MR imaging predict malignant transformation? Radiology 247(1):170-178. https://doi.org/10. 1148/radiol.2471062089

16. Hakyemez B, Erdogan C, Ercan I, Ergin N, Uysal S, Atahan S (2005) Highgrade and low-grade gliomas: differentiation by using perfusion MR imaging. Clin Radiol 60(4):493-502. https://doi.org/10.1016/j.crad.2004.09.009 
17. Sugahara T, Korogi Y, Tomiguchi S, Shigematsu Y, Ikushima I, Kira T, Liang L, Ushio Y, Takahashi M (2000) Posttherapeutic intraaxial brain tumor: the value of perfusion-sensitive contrast-enhanced MR imaging for differentiating tumor recurrence from nonneoplastic contrast-enhancing tissue. AJNR Am J Neuroradiol 21(5):901-909

18. Warmuth C, Gunther M, Zimmer C (2003) Quantification of blood flow in brain tumors: comparison of arterial spin labeling and dynamic susceptibility-weighted contrast-enhanced MR imaging. Radiology 228(2): 523-532. https://doi.org/10.1148/radiol.2282020409

19. Brendle C, Hempel JM, Schittenhelm J, Skardelly M, Tabatabai G, Bender B, Ernemann U, Klose U (2017) Glioma grading and determination of IDH mutation status and ATRX loss by DCE and ASL perfusion. Clin Neuroradiol. https://doi.org/10.1007/s00062-017-0590-z

20. Law M, Young R, Babb J, Rad M, Sasaki T, Zagzag D, Johnson G (2006) Comparing perfusion metrics obtained from a single compartment versus pharmacokinetic modeling methods using dynamic susceptibility contrastenhanced perfusion MR imaging with glioma grade. AJNR Am J

Neuroradiol 27(9):1975-1982

\section{Publisher's Note}

Springer Nature remains neutral with regard to jurisdictional claims in published maps and institutional affiliations.

\section{Submit your manuscript to a SpringerOpen ${ }^{\circ}$ journal and benefit from:}

- Convenient online submission

- Rigorous peer review

- Open access: articles freely available online

High visibility within the field

- Retaining the copyright to your article

Submit your next manuscript at $\boldsymbol{\nabla}$ springeropen.com 